\title{
“NI HABLAR!”. ESTUDIO CONTRASTIVO DE DOS FUNCIONES COMUNICATIVAS OPUESTAS EN LAS VARIEDADES PENINSULAR Y RIOPLATENSE DEL ESPAÑOL ACTUAL
}

\author{
ANAIS HOLGADO LAGE \\ Princeton University \\ EDGARDO GUSTAVO ROJAS \\ CEIL-IDIHCS-FAHCE-Universidad Nacional de La Plata
}

\section{RESUMEN}

Este artículo compara las funciones pragmáticas que desempeña el marcador discursivo ni hablar en las variedades peninsular y rioplatense del español. Para ello, llevamos a cabo un experimento con hablantes nativos de los dos dialectos, con preguntas sobre su propio uso de la expresión. Los resultados muestran que esta partícula, empleada de forma normalmente reactiva ante lo pronunciado por un interlocutor, puede comunicar actitudes de polaridad contraria ante enunciados declarativos y directivos: en el español peninsular expresa desacuerdo o rechazo frente a lo dicho, mientras que en la variedad rioplatense indica aceptación, en ambos casos con rotundidad. Dado que la bibliografía especializada no refleja este contraste de uso entre los diferentes dialectos, nuestro trabajo pretende contribuir de forma relevante al estudio de los marcadores conversacionales en el estado actual de la lengua española.

PALABRAS CLAVE: marcadores discursivos, funciones comunicativas, acuerdo, rechazo, variedad rioplatense, variedad peninsular.

\section{ABSTRACT}

This article compares the pragmatic functions performed by the discourse marker ni hablar from peninsular and Río de la Plata Spanish. In order to do that, we conducted an experiment with native speakers of both dialects who answered questions about their own use of the expression. Results show that this particle, normally used as a reaction to what the interlocutor has said, can communicate contrary attitudes after declarative or directive statements; in peninsular Spanish it expresses disagreement or rejection to what has been said, while in Río de la Plata Spanish it shows acceptance, in both cases categorically. As previous research has not uncovered this contrast of use between the dialects, 
our work aims to offer a relevant contribution to the study of conversational discourse markers in the current state of the Spanish language.

KEYWORDS: discourse markers, communicative functions, agreement, disagreement, Río de la Plata Spanish, peninsular Spanish.

\section{INTRODUCCIÓN}

Los marcadores discursivos fueron durante mucho tiempo ignorados dentro de la investigación lingüística en español. Como ya señalaba Vázquez Veiga (1994-1995: 349), "a pesar de la importante labor que desempeñan dentro de la estructura de un texto, podemos afirmar que, en general, la tradición española [...] ha mostrado un interés muy escaso por el estudio de los marcadores".

Sin embargo, en las dos últimas décadas se han multiplicado los estudios sobre marcadores del discurso, como se puede comprobar por la gran cantidad de trabajos de reciente publicación ${ }^{1}$. Una de las tendencias actuales de investigación incluye estudios sobre grupos de marcadores a partir de una clase particular de palabra. Es el caso de obras como las de Montes (1999), que estudia las conjunciones, y Garcés Gómez (2013), que se ocupa de los adverbios con función discursiva. También con frecuencia se analizan grupos de marcadores para expresar una función determinada: por ejemplo, Acín Villa (2000), sobre los "digresores"; Domínguez García (2002a), que se refiere al amplio grupo de los "conectores"; San Martín Núñez (2013), que analiza los "reformuladores de distanciamiento" o Vázquez Veiga (2003), sobre los “marcadores de recepción”.

Adquieren especial relevancia para nuestro estudio sobre el marcador ni hablar los estudios monográficos sobre estos elementos, especialmente los polifuncionales. En este aspecto, algunos marcadores han sido ampliamente estudiados: pues se analiza en Portolés (1989), Domínguez García (2002b), Hernando Cuadrado (1994), Sánchez Carranza (2013) o Uribe Mallarino (2005). En la mayor parte de los casos, estos marcadores, que presentan cierta variedad en sus funciones ${ }^{2}$, se analizan desde un punto de vista puramente pragmático. Además, el número de elementos ampliamente estudiados es muy pequeño en comparación con la cantidad de

${ }^{1}$ Se incluyen libros especializados como Martín Zorraquino y Montolío Durán (coords.) (1998), Loureda y Acín Villa (eds.) (2010), Aschenberg y Loureda (eds.) (2011), González Ruiz y Llamas Saíz (eds.) (2011).

${ }^{2}$ Pese a que un alto número de marcadores son polifuncionales, no se produce con frecuencia el alto contraste entre funciones que se verá más adelante en este trabajo, ya que hallamos significados antónimos para el mismo marcador. 
marcadores discursivos que existen en nuestra lengua. Por todo ello, todavía queda mucho camino por recorrer y una gran número de marcadores susceptibles de ser estudiados en profundidad.

En cualquier caso, no se puede negar que estos elementos tan necesarios para la comunicación han suscitado un interés reciente en el mundo de la investigación. Este hecho ha ayudado a establecer cierta homogeneidad con respecto a la definición de los marcadores, considerados "elementos normalmente de escaso cuerpo fonético, invariables en la mayoría de los casos, que unen oraciones a la vez que expresan relaciones entre ellas, y que poseen relevancia en el discurso" (Holgado Lage, 2014: 16-17). No obstante, podemos afirmar que esta esfera de la lengua todavía presenta unos límites difusos, y no está claro entre los estudiosos cuáles son los elementos que integran este grupo. Martín Zorraquino (2011: 845-846) concluye que cada autor incluye y excluye diferentes elementos siguiendo su propia concepción de lo que constituye un marcador discursivo. Así, ciertos elementos aparecen en algunos estudios sobre marcadores y en otros no. A título de ejemplo, el Diccionario de conectores y operadores del español (Fuentes Rodríguez, 2009) incluye numerosas expresiones como hablando en plata o gracias a Dios, que no aparecen registradas en otras fuentes, como el Diccionario de partículas discursivas del español ${ }^{3}$ (Briz, Pons Bordería y Portolés, 2004).

En concreto, el marcador que nos ocupa, ni hablar, aparece registrado en Fuentes Rodríguez y también en el Diccionario de partículas (Santos Río, 2003), pero no en el Diccionario de partículas discursivas del español. Por otra parte, algunos estudios de marcadores sí se refieren a esta expresión en concreto, pero, tal y como veremos a continuación, aparece catalogada en prácticamente todos los casos como expresión de negación o rechazo, cuando no es ese su único uso.

\section{EsTAdO DE LA CUESTIÓN SOBRE EL MARCADOR NI HABLAR}

\subsection{Ni hablar en tratados sobre marcadores discursivos}

Si los marcadores del discurso no han acaparado la atención de los lingüistas hasta las últimas décadas del siglo pasado, menos frecuente ha sido el tratamiento de aquellos que se emplean preferentemente -cuando no exclusivamente- en el registro informal y oral de la lengua. Tal circunstancia obedece, principalmente, a las dificultades que acarrea la catego-

\footnotetext{
${ }^{3}$ Este diccionario puede consultarse online en $<$ www.dpde.es $>$.
} 
rización de estas expresiones por su heterogeneidad, polifuncionalidad e inestabilidad diacrónica, características estudiadas por diversos autores ${ }^{4}, \mathrm{y}$ que se aprecian especialmente cuando se trata de marcadores eminentemente conversacionales (Portolés, 1998; Rojas, 2011a, 2011b y 2012). El caso de ni hablar es particularmente significativo, ya que, en comparación con otras locuciones habituales en español que adoptan la pauta $n i+$ infinitivo, esta se ha fijado muy recientemente, en el siglo Xx (Pérez-Salazar, 2009: 40), y ostenta un alto grado de informalidad y recurrencia en el habla cotidiana. Algunas de estas expresiones formadas con $n i$ llegan incluso a calificarse de malsonantes (RAE, 2010: 943), aunque no es el caso de ni hablar.

Según los estudios pragmáticos consultados, ni hablar es un marcador empleado principalmente en contextos dialogados, conversacionales y espontáneos para expresar negación, desacuerdo y rechazo. Por ejemplo, Casamiglia y Tusón Valls (1999: 239) lo incluyen en un grupo llamado "marcadores de desacuerdo", Mancera Rueda (2009: 154) lo llama "marcador de rechazo" y Landone (2009: 236-287) indica que este marcador "no admite, consiente o confirma". En el diccionario de Fuentes Rodríguez (2009: 226) se resalta que esta locución "indica rechazo rotundo a una expresión (opinión, mandato, petición) anterior de otro interlocutor" ${ }^{5}$. Se trata, por consiguiente, de un marcador del discurso empleado de forma reactiva ante enunciados declarativos o directivos producidos normalmente por un interlocutor en el intercambio coloquial, aunque, como indica PérezSalazar (2009: 51) y como veremos en los resultados de nuestro experimento, también puede aparecer en pasajes monologados. Cabe aclarar, no obstante, que estos trabajos registran mayoritariamente los usos del marcador en el español peninsular, y que no identifican significados diferentes en otras variedades lingüísticas ${ }^{6}$, con la excepción de Pérez-Salazar (2009: 52), que menciona un "valor escasamente documentado de ni hablar en sentido positivo", encontrado en Uruguay, y que, como comprobaremos en el próximo apartado, está muy extendido en la zona rioplatense de Argentina. En Holgado Lage (2014: 350) también se menciona brevemente este uso de aceptación ante lo dicho.

A diferencia de otros marcadores conversacionales de aceptación o acuerdo frente a lo dicho, ni hablar es un marcador que, en su función más

${ }^{4}$ Véanse por ejemplo Martin Zorraquino y Portolés (1999), Martín Zorraquino (2004), Portolés (2008) o Garcés Gómez (2008).

${ }^{5}$ Similares categorizaciones se dan en otros autores como Perona (2000) y Pérez-Salazar (2009)

${ }^{6}$ Pese a que esto es bastante habitual, recientemente encontramos una mayor bibliografía referida específicamente a usos de marcadores en otras zonas del territorio hispanohablante, por ejemplo San Martín Núñez (2013), Sánchez Carranza (2013) o Uribe Mallarino (2005). 
extendida, supone una situación de conflicto con la cortesía verbal ${ }^{7}$. Esto se debe a que este marcador señala, de forma categórica, el grado máximo de rechazo y desacuerdo; de hecho, entre los marcadores que los autores consultados clasifican en el mismo grupo que ni hablar, los más frecuentes resultan ser en absoluto y de ningún/a modo/manera/forma (RAE, 2010: 943), expresiones que también comunican un desacuerdo rotundo o un rechazo absoluto. La diferencia es que estas últimas expresiones se consideran formales, mientras que ni hablar, como ya hemos mencionado, se cataloga como informal (Pérez-Salazar, 2009: 38-39).

\subsection{Ni hablar en gramáticas y diccionarios académicos}

Las últimas versiones del Diccionario de la Lengua Española (RAE, 2001 y 2014) incluyen esta locución en la entrada lexicográfica hablar, donde se presenta como una expresión empleada para "rechazar o negar una propuesta", lo que parece indicar que estos diccionarios limitan el uso de $n i$ hablar a los contextos dialogados cuyo primer turno de habla viene dado por un enunciado directivo. Asimismo, conviene señalar que estos diccionarios presentan la expresión ni hablar del peluquín como una variante del marcador discursivo que analizamos en este trabajo y que ha sido empleada en nuestro estudio por varios informantes.

No encontramos referencias a este marcador discursivo -ni ejemplos que lo incluyan- en el Diccionario panhispánico de dudas (RAE, 2005) ${ }^{8}$, y tampoco se menciona en la Gramática de referencia para la enseñanza de español (Borrego Nieto, 2013), donde aparece un apartado para "expresar aceptación o acuerdo" pero no para expresar lo contrario. La Nueva gramática de la lengua española (RAE, 2010: 632) lo incluye como locución interjectiva de "negación, rechazo u oposición”, aunque también lo menciona bajo los epígrafes de "expresión exclamativa" y "locución negativa”. No hay que olvidar que las obras de este tipo se ciñen preferentemente a las categorías gramaticales y se enfrentan con el problema de la heterogeneidad y diversidad -tanto formal como funcional- que caracterizan a los marcadores discursivos.

Entre las gramáticas de referencia académica más extendidas y consultadas, el capítulo más amplio y detallado sobre marcadores del discurso

\footnotetext{
${ }^{7}$ Es decir, aquella que apela al deseo de aceptación, el sentido de pertenencia y las necesidades gregarias de los hablantes (Brown y Levinson, 1978; Haverkate, 1994; Escandell Vidal, 1996).

${ }^{8}$ Quizás esto se deba a que la única función reconocida del marcador es la de negación o rechazo y por tanto no tiene por qué plantear dudas en el hablante.
} 
es el de Martín Zorraquino y Portolés (1999), incluido en la Gramática descriptiva de la lengua española. Se trata de una aproximación pragmática que -inspirada en la teoría de la relevancia9- caracteriza a los marcadores del discurso por su significado procedimental, considerando que estos han perdido su sentido conceptual originario como resultado de un proceso de gramaticalización, para especializarse en aportar instrucciones sobre el procesamiento cognitivo del discurso. Dicho capítulo propone una clasificación ampliamente consensuada y extendida en los estudios pragmalingüísticos sobre marcadores discursivos que consta de cinco grupos: estructuradores de la información, conectores, reformuladores, operadores argumentativos y marcadores conversacionales. Estos últimos incluyen una subclase "de modalidad epistémica" y, dentro de esta, otra llamada "de evidencia" en la que se mencionan marcadores como sí y $n o^{10}$.

\subsection{Ni hablar en los corpus de la lengua española}

Los corpus académicos en lengua española de mayor consulta ofrecen resultados dispares en el registro del marcador analizado. Así, el Corpus de Referencia del Español Actual (RAE, banco de datos CREA) arroja como resultado para ;Ni hablar! -literal, como construcción independiente, entre signos de exclamación- un total de 200 registros, 145 de los cuales -el $72,5 \%$ del total- corresponden a España, en tanto que 5 registros -es decir, el 2,5\% de la muestra- corresponden a Argentina. La misma búsqueda en el Corpus Diacrónico del Español (RAE, banco de datos CORDE) resulta menos productiva, ya que ofrece un total de 40 registros, en similar proporción: treinta fragmentos corresponden a España -el $75 \%$ de los casos-y un único registro -que representa también el 2,5\% del total-corresponde a Argentina, lo que es lógico teniendo en cuenta que, como ya se ha señalado, ni hablar es una expresión de reciente gramaticalización.

Si bien nuestro objetivo no es analizar las apariciones del marcador $n i$ hablar en estos bancos de datos, este resumen de resultados constituye un indicador significativo de la desproporción que se encuentra en los registros disponibles en línea a la hora de realizar un estudio comparativo de su empleo en las dos variedades consideradas. Por otra parte, los escasos

${ }^{9}$ Esta es la orientación que también adopta Portolés (1998) en una obra plenamente dedicada al estudio de los marcadores del discurso. En las últimas décadas, la teoría de la relevancia, formulada por Sperber y Wilson (1986) y aplicada por Blakemore (1988-1992) a la cohesión textual, ha sustentado buena parte de los estudios pragmalingüísticos sobre marcadores del discurso.

${ }^{10}$ Si bien ni hablar no aparece descrito en la obra, creemos que vale la pena mencionarla, tanto por su importancia en el estudio de los marcadores discursivos como por la clara relación entre la última categoría referida y la expresión que analizamos en este artículo. 
registros de iNi hablar! en el CREA y en el CORDE para Argentina no permiten distinguir a qué variedad del español, entre las registradas en este país, corresponde cada uno de los casos, lo que no es útil para este estudio, que se centra principalmente en el español rioplatense.

Por todo ello, hemos realizado una consulta en el corpus en línea más reciente, el Corpus del Español del Siglo XXI (RAE, banco de datos CORPES XXI). En él, hemos recuperado un total de 190 ejemplos con origen en España de ni hablar -literal- en todos los campos admitidos (medios, soportes, temas y tipologías). El análisis de dichos resultados indica que en 84 casos -cifra que representa el $44.21 \%$ del total- la secuencia referida aparece en forma independiente, enmarcada entre signos de puntuación o exclamación, o como único componente de un turno de habla completo $^{11}$-criterios que permiten identificar su empleo como marcador-, mientras que en los registros restantes el grupo ni hablar se encuentra integrado en la oración. En general, en los fragmentos donde esta locución funciona como marcador presenta un significado de negación, desacuerdo o rechazo, en sintonía con la bibliografía consultada.

Siguiendo el mismo criterio de búsqueda, este corpus recupera un total de 156 registros con origen en Argentina. Aunque, en comparación con los resultados obtenidos en CORDE y CREA, este valor se acerca más a los resultados de la variedad peninsular, solo 26 fragmentos recuperados -que representan un $16,6 \%$ del total- incluyen ni hablar desligado del contexto oracional y enmarcado entre signos de puntuación o de exclamación. En definitiva, si bien este corpus académico ofrece una muestra más actualizada de registros, y una menor diferencia en el resultado absoluto de la búsqueda para ambas variedades del español, los valores relativos para los usos extraoracionales y fraseológicos de ni hablar también resultan desproporcionados y poco funcionales a fines comparativos, aunque es posible que esto se deba a que, según indican nuestros datos, la expresión se usa de forma más habitual en el territorio peninsular.

\section{Metodología y ANÁlisis de los datos}

\subsection{Metodología utilizada}

En vista de los resultados expuestos en el apartado anterior, analizamos el uso de ni hablar como negación rotunda propio de la variedad peninsular y de otras comunidades hispanohablantes, ampliamente registrado,

\footnotetext{
${ }^{11}$ Son principalmente las obras enfocadas en la pragmática las que destacan el carácter independiente, marginal o parentético de los marcadores del discurso, es decir, su independencia sintáctica (Portolés, 1998; Martín Zorraquino y Portolés, 1999).
} 
estudiado y extendido, y también la función que, según nuestros datos, es en la actualidad más habitual en la provincia de Buenos Aires y territorios adyacentes, donde se emplea como aceptación o refuerzo positivo ante lo que acaba de decirse. Con el objetivo de reunir datos de hablantes sobre su uso de la expresión ni hablar, llevamos a cabo un experimento con 90 hablantes nativos de ambas variedades lingüísticas, adultos -entre los 18 y los 60 años de edad- y con estudios secundarios finalizados. Las encuestas se dividieron según el origen de los participantes: 50 se administraron a hablantes argentinos y 40 a hablantes españoles.

Para la recolección de los datos, diseñamos un prototipo de encuesta donde se pedía a los usuarios que respondieran a ciertas preguntas sobre esta expresión y su empleo, en primer lugar, la frecuencia de utilización del marcador. Aunque en muchas ocasiones los hablantes no tienen una concepción real del uso propio de una expresión, esta pregunta sirvió para analizar la familiaridad de los encuestados con el marcador. También se les preguntó con qué contextos asocian la expresión ni hablar, con el objetivo de comparar si en ambas comunidades lingüísticas se utiliza en las mismas situaciones comunicativas. A continuación, pedimos a los participantes que escribieran una pequeña definición del marcador. En este apartado, dado que las funciones estudiadas son opuestas, se pudo ver claramente la diferencia entre la utilización como expresión de rechazo o negación y la de aceptación ante lo dicho. Finalmente, los encuestados debían aportar un ejemplo donde se utiliza el marcador ni hablar a partir de su experiencia e intuición como hablantes nativos de español.

\subsection{El uso de ni hablar por hablantes de español peninsular}

Según los datos recolectados, el $88 \%$ de nuestros informantes españoles afirma conocer la expresión ni hablar, el $68 \%$ la emplea ocasionalmente y el $20 \%$ de los encuestados indica que la usa habitualmente. Si tenemos en cuenta las explicaciones, expresiones equivalentes o ejemplos aportados en cada caso, el $88 \%$ de los encuestados asigna a ni hablar un valor negativo. Es decir, ningún encuestado propuso un valor diferente. El carácter negativo de la expresión aparece remarcado en el $38 \%$ de los ejemplos propuestos por los informantes a través de otras expresiones contiguas -sobre todo, los adverbios no y jamás-, como puede apreciarse en las siguientes respuestas extraídas de las encuestas:

(1) A: ¿Pero no vas a prestarle dinero?

B: No, ni hablar, no se me ocurriría jamás hacerlo.

(2) Ni hablar, no pienso hacer eso que me pides. 
En el $65 \%$ de las encuestas correspondientes a este grupo de hispanohablantes se señala que el rechazo o la negación expresado por el marcador es absoluto; los informantes indican que el valor negativo del marcador se expresa de forma total, tajante, enfática, completa y categórica; también afirman que el uso del marcador supone el fin de una discusión y que no deja lugar a argumentar lo contrario. Tal y como indica PérezSalazar (2009), los informantes remarcan en el 22\% de los casos el carácter coloquial o informal del marcador discursivo. A continuación se muestran algunas respuestas ilustrativas para cada una de estas dos coincidencias: su carácter absoluto, en los ejemplos (3) y (4), e informal, en los ejemplos (5) y (6):

(3) Es una expresión que denota un "no" rotundo absoluto ante una sugerencia o una petición de permiso.

(4) Expresión que supone una negativa rotunda ante un hecho, una petición, una sugerencia, etcétera.

(5) En contextos informales, con personas con las que tengo confianza, de otro modo puede significar que quiero poner distancia.

(6) En una situación informal. En una conversación con amigos.

En la tarea en la que los informantes deben proponer expresiones sinónimas, hemos hallado diferencias respecto a la bibliografía consultada. En absoluto y de ningún/a modo/manera ${ }^{12}$, registradas a menudo en los estudios sobre marcadores (Perona, 2000; Landone, 2009) como expresiones sinonímicas de ni hablar, solo fueron seleccionadas, respectivamente, por el $10 \%$ y por el $22 \%$ de los encuestados. Por el contrario, el 35\% eligió la expresión ni de coña, más próxima a la estudiada por su uso informal. Fueron también frecuentes otras expresiones análogas que se forman a partir de la conjunción ni, tales como ni pensarlo, ni loco, ni mucho menos, ni de broma, ni de lejos, ni de casualidad, ni lo sueñes, ni una mierda, llegando a aparecer al menos una de ellas en el $50 \%$ de las encuestas administradas a este grupo. Del mismo modo, se registra en el $40 \%$ de las encuestas ${ }^{13}$ alguno de los distintos marcadores con nada, tales como para nada, de eso nada y nada de nada. En el 10\% de las encuestas, aparece la locución ni hablar del peluquín, actualmente considerada por la RAE (2014) como una

${ }^{12}$ La expresión de ninguna forma, habitual en la variedad rioplatense, no se registró entre los hablantes españoles.

${ }^{13}$ Dado que los encuestados podían incluir tantos marcadores sinonímicos como quisieran, el número, al sumar los porcentajes, puede ser superior a 100. 
expresión sinonímica de ni hablar. Se indica en las respuestas de los participantes que es una variante en desuso, equivalente o complementaria, tal y como muestran los siguientes ejemplos:

(7) Se usa para negar tajantemente como "ni de coña", "ni en broma". A veces se complementa con "ni hablar del peluquín”.

(8) Ni de coña.

Ni mijita.

Ni hablar del peluquín (alguna vez se lo he oído a mi madre, jaja).

No.

En el $20 \%$ de las encuestas se ejemplifica el uso del marcador ni hablar como enunciado completo en un único turno de habla, lo que constituye un rasgo distintivo de los marcadores conversacionales. Además, indica su alto nivel de gramaticalización en el estado actual del español peninsular ${ }^{14}$ :

(9) A: ¿Subimos los 30 pisos por la escalera?

B: Ni hablar.

(10) A: ¿Hoy no te quedas haciendo horas extra?

B: ¡Ni hablar!

El $48 \%$ de los informantes propone ejemplos donde el marcador aparece en un diálogo, que normalmente incluye una petición, invitación o propuesta seguida de una respuesta de rechazo, como en los ejemplos anteriores. Sin embargo, en el 38\% de los casos esta expresión aparece de forma autorreactiva. En muchas ocasiones se sugiere que ha habido un enunciado anterior que se rechaza, como en los ejemplos (11), (12) y (13), y en otras ocasiones no parece haber un enunciado previo, como es el caso de (14). En estos casos monologados el marcador no siempre aparece al comienzo de la intervención, sino que puede aparecer intercalado en el texto y en ocasiones desplazado hasta el final del enunciado. La movilidad es otra característica que comparten la mayoría de los marcadores discursivos.

(11) ¿Que te preste cien euros? Ni hablar.

(12) ¿Trabajar sesenta horas semanales por cien euros al mes? Ni hablar.

(13) Ni hablar, no voy a hacer eso que me pides.

(14) Este verano no me quedo en casa, ni hablar.

\footnotetext{
${ }^{14}$ Pese a que la reduplicación se ha presentado como otra de las características de los marcadores conversacionales (Portolés, 1998), no se han hallado ejemplos de este proceso en las encuestas.
} 
En los casos en que ni hablar aparece en un diálogo, se muestra una clara tendencia a su uso después de una pregunta, petición o invitación, tal y como muestran los ejemplos (15) y (16), mientras que no son especialmente frecuentes los usos como respuesta a una aserción, como en el ejemplo (17).

(15) A: Mañana tienes que levantarte a las 5 para poner una lavadora.

B: ¡Ni hablar! No pienso hacerlo.

(16) A: Papá, ¿me compras un perro?

B: Ni hablar, si quieres vamos a la protectora y adoptamos uno.

(17) A: Los profesores de Cursos Internacionales están tremendamente formados y son muy competentes en su trabajo.

B: Ni hablar. No tienen ni idea...

A pesar de que la bibliografía consultada indica a menudo que ni hablar se emplea tanto para manifestar rechazo ante un enunciado directivo como para comunicar desacuerdo ante un enunciado declarativo (Fuentes Rodríguez, 2009), solamente el $4 \%$ de nuestros informantes ha seleccionado este último empleo.

\subsection{El uso de ni hablar por hablantes de español rioplatense}

En el caso del uso de este marcador por parte de los hablantes de español rioplatense hallamos grandes diferencias, no solo con respecto a las encuestas de los hablantes de español peninsular sino también respecto a lo señalado en la bibliografía consultada. En primer lugar, encontramos que el $44 \%$ de los encuestados afirma utilizarla con uso ocasional, y el $34 \%$ con uso habitual; el resto indica que normalmente no utiliza esta expresión, con lo cual parece que se usa con más frecuencia en España que en la zona de la provincia de Buenos Aires. Sin embargo, la mayor diferencia aparece en el valor asignado a esta expresión, ya que un $82 \%$ de los participantes le asigna un valor positivo, de refuerzo ante lo dicho por el interlocutor y en muchos casos se resalta que lo que se ha dicho es algo obvio, que no admite discusión. Podemos apreciar este tipo de definiciones aportadas por los encuestados en (18), (19) y (20):

(18) Sirve para explicar que no hace falta aclarar más, algo que se da por entendido.

(19) Algo que se da por obvio, no hace falta explicarlo o decirlo.

(20) No hace falta hablar de algo en particular, ya que es evidente. 
Esto se comprueba claramente en los ejemplos dados por los participantes en los que aparecen marcadores que refuerzan lo que se está intentando expresar, ya que de otro modo los ejemplos pueden resultar ambiguos. Se utiliza a menudo el marcador sí, concretamente en un $14 \%$ de los casos, como en los siguientes ejemplos:

(21) A: Creo que habría que terminar con la violencia.

B: Sí, ni hablar.

(22) A: ¿Viste qué bien toca el piano?

B: Sí, ni hablar. Es una genia.

No obstante, encontramos que en un $6 \%$ de las encuestas se habla del valor negativo de este marcador, y solamente en un $2 \%$ se mencionan los dos valores:

(23) Expresión usada con tono exclamativo para rehusar o negar.

(24) Tiene un carácter ambiguo. Puede negar o reafirmar una situación.

Esta información se puede resumir en el siguiente gráfico comparativo de las encuestas de ambos grupos (fig. 1):

\section{FIGURA 1.}

\begin{tabular}{|l|c|c|c|c|}
\cline { 2 - 5 } \multicolumn{1}{c|}{} & Valor negativo & Valor afirmativo & Ambos valores & No responde \\
\hline España & $88 \%$ & $0 \%$ & $0 \%$ & $12 \%$ \\
\hline Argentina & $6 \%$ & $82 \%$ & $2 \%$ & $10 \%$ \\
\hline
\end{tabular}

Podemos afirmar que el valor afirmativo de la expresión no está tan fijado como el negativo en el territorio peninsular, ya que, aunque el porcentaje es pequeño, parece que ciertos hablantes de español rioplatense sí conocen el uso contrario tan popular en otras zonas hispanohablantes, algo que no sucede a la inversa. $\mathrm{Al}$ igual que en el caso de las encuestas de la zona española, el valor que se le da a ni hablar es categórico, evidente y absoluto. El 30\% además añade que lo utiliza en contextos informales o con amigos:

(25) En charlas con gente que conozco.

(26) En ámbitos informales. 
Como es lógico, las expresiones sinonímicas son en su gran mayoría diferentes. Solamente un $4 \%$ menciona de ninguna manera, lo que se corresponde con el porcentaje de encuestados que aluden al valor negativo del marcador. Los marcadores que aparecen con más frecuencia en estas encuestas son obvio/obviamente (el 26\% de las encuestas lo incluye), seguro $(12 \%)$, si $(10 \%)$, claro $(10 \%)$, por supuesto $(6 \%)$, y expresiones a partir del verbo dudar (12\%), como no lo dudes, ni dudarlo, sin dudas o sin ninguna duda. Como es lógico, estos marcadores no aparecen en las fuentes consultadas como sinónimos de ni hablar, dado que el uso afirmativo del marcador no se registra generalmente en los estudios sobre marcadores ni en las gramáticas académicas.

En cuanto al uso discursivo, el 74\% de los encuestados da ejemplos en los que ni hablar aparece excluido de la predicación, lo que parece indicar que el uso como marcador discursivo está bastante fijado en la mente de los hablantes de esta zona, un porcentaje muy similar al que se producía entre los hablantes del territorio peninsular. Encontramos también, como en el otro grupo de encuestas, un gran número de ejemplos producidos por los participantes donde se representa una situación de diálogo, con un turno de habla que comienza con un marcador de reafirmación, en la mayoría de los casos el mismo ni hablar, en otros, como ya hemos visto, sí, como se muestra en (27). En un $34 \%$ de las encuestas, ni hablar aparece acaparando todo el turno de habla, como se ve en (28), un número bastante más alto que el $20 \%$ de las encuestas de España, pero no lo suficiente como para mostrar una clara diferencia en el uso del marcador. No obstante, solamente encontramos un $6 \%$ de los casos donde ni hablar aparece en un contexto monologado, reaccionando ante algo que se ha dicho antes pero no aparece especificado, como en (29) o donde no parece hacer referencia necesariamente a algo que se ha dicho antes -ejemplo (30)-, sino que puede estar reaccionando ante una acción.

(27) A: ¡Es tedioso tener que hacer este práctico!

B: Sí, ni hablar.

(28) A: $2+2$ es 4 .

B: ¡Ni hablar!

(29) Sí, ni hablar, no me quedan dudas que tal persona...

(30) Todos somos buenos alumnos, ni hablar.

Este hecho, si bien coincidente con las fuentes analizadas en las que se indica que este es un marcador reactivo ante lo dicho por el interlocutor 
(Fuentes Rodríguez, 2009; Perona, 2000) ${ }^{15}$, difiere mucho del de las encuestas del territorio español, donde inesperadamente un $38 \%$ de los casos son autorreactivos ${ }^{16}$. Parece que en el caso de España el marcador se está extendiendo más rápidamente de su uso tradicional en el diálogo a otros contextos, como se puede observar en este cuadro resumen (fig. 2):

Figura 2.

\begin{tabular}{|l|l|c|}
\hline \multirow{2}{*}{ España } & Ejemplo dialogado & $48 \%$ \\
\cline { 2 - 3 } & Ejemplo monologado & $38 \%$ \\
\hline \multirow{2}{*}{ Argentina } & Ejemplo dialogado & $60 \%$ \\
\cline { 2 - 3 } & Ejemplo monologado & $6 \%$ \\
\hline
\end{tabular}

El último factor a tener en cuenta para la comparación de las encuestas es la preferencia por un tipo concreto de acto de habla anterior, es decir, si el marcador ni hablar reacciona ante una aserción o ante una petición, pregunta o sugerencia. En el caso del primer grupo de encuestas analizadas, claramente el segundo contexto era el preferido por los participantes, con mucha diferencia. En el caso de los hablantes argentinos, los resultados muestran que ambas reacciones, ante un acto declarativo o directivo, son susceptibles de ir seguidas por este marcador; así, en un $28 \%$ de los ejemplos dados, ni hablar se emplea para reaccionar positivamente ante una afirmación del interlocutor, como puede apreciarse en (31) y (32), y en un $24 \%$, para reaccionar favorablemente ante una pregunta o petición, como se muestra en (33) y $(34)^{17}$.

(31) A: Borges es un ícono de la literatura.

B: ¡Ni hablar!

(32) A: Necesitamos un aumento de sueldo porque...

B: iNi hablar!

15 No coincidente del todo, ya que la bibliografía se refiere exclusivamente a su uso como negación.

${ }^{16}$ Aunque no podemos olvidar que en muchos casos los ejemplos parecían responder a algo que se había dicho antes pero que no aparecía registrado.

${ }_{17}$ A pesar de que estos últimos cuatro ejemplos pueden parecer ambiguos, sabemos que el participante quería expresar un valor afirmativo a partir del contenido del resto de su encuesta. 
(33) A: ¿Le digo a Gus que trabaje mejor?

B: ¡Ni hablar!

(34) A: ¿Jugás al fútbol?

B: Ni hablar.

De nuevo, por tanto, el uso de este marcador en el español rioplatense se asemeja más a lo indicado en algunas de las fuentes consultadas en cuanto a su carácter reactivo ante afirmaciones o peticiones, si bien no deja de resultar curioso que la función pragmática del marcador es en la inmensa mayoría de los casos contraria a la registrada en dicha bibliografía.

\section{Conclusiones}

Ni hablar es un marcador del discurso -en concreto, un operador (Fuentes Rodríguez, 2009) - que se utiliza actualmente para emplear funciones diferentes en las variedades peninsular y rioplatense del español: en la primera, se utiliza para negar; en la segunda, se emplea para reafirmar algo. Esta reacción es en ambos casos tajante, no deja lugar a dudas, y se utiliza principalmente en el registro informal o coloquial de las dos variedades lingüísticas. Mientras que las fuentes y los corpus consultados hacen referencia a su función prototípica de negación o rechazo ${ }^{18}$, los resultados de nuestra investigación indican que en la provincia de Buenos Aires la función más conocida y extendida es precisamente la contraria.

Nuestro estudio consistió en una encuesta administrada a 90 participantes de ambas variedades lingüísticas en la que se les preguntó sobre su percepción del marcador. Los resultados sugieren que, mientras que en España se conoce y se produce exclusivamente la función de rechazo o negación, entre los participantes de Argentina la función predominante es la de aceptación o de remarcación de algo que es considerado obvio. Este uso, escasamente registrado hasta la fecha, merece ser tenido en cuenta por su amplio reconocimiento en esta zona hispanohablante. Las definiciones producidas por los encuestados, las expresiones que consideran equivalentes y los ejemplos que aportan confirman la idea de afirmación. Esta oposición de significados constituye un fenómeno peculiar en el ámbito de los marcadores del discurso, donde en muchas ocasiones un marcador se utiliza para diferentes funciones (véase, por ejemplo, el polifacético pues), pero no es habitual que estas funciones sean tan diferentes entre sí.

${ }^{18}$ Con la excepción de una pequeña nota de Pérez Salazar (2009) sobre un posible uso positivo en el Río de la Plata. 
En cuanto a los contextos de uso preferidos por los hablantes, los datos reflejan que ni hablar no se usa solamente como expresión reactiva, que es el contexto mencionado en la mayoría de las fuentes consultadas, sino que, especialmente en el español peninsular, puede aparecer en el monólogo, a modo de marcador autorreactivo, como señala Pérez Salazar (2009).

Se produce, además, una diferencia importante entre ambos grupos en la preferencia sobre el acto de habla que produce la reacción. Los hablantes de español peninsular lo emplean en su gran mayoría ante enunciados directivos -peticiones, invitaciones, sugerencias o preguntas-, mientras que los hablantes de español rioplatense lo utilizan con frecuencia también para manifestar acuerdo frente a una afirmación declarativa del interlocutor.

Este trabajo acerca de las funciones opuestas de ni hablar pretende ser una primera aproximación teórica y experimental que abre nuevas líneas de investigación para el estudio de este marcador discursivo. Así, deberá comprobarse si su uso para expresar acuerdo se extiende más allá del área rioplatense o hasta qué punto los hablantes de dicha variedad conocen el uso prototípico de rechazo o negación. Esta contribución proporciona nuevos enfoques en la comprensión del funcionamiento de los marcadores discursivos a través de la variación diatópica, así como en el análisis del discurso oral en español. 


\section{BIBLIOGRAFÍA}

Acín Villa, E. (2000): "Por cierto, a propósito y otros digresivos", en P. Carbonero Cano et al. (eds.), Lengua y discurso. Estudios dedicados al profesor Vidal Lamíquiz, Madrid, Arco/Libros, págs. 59-72.

Aschenberg, H. y Loureda, O. (eds.) (2011): Marcadores del discurso: de la descripción a la definición, Madrid/Frankfurt, Iberoamericana/Vervuert.

BLAKemore, D. (1988-1992): "La organización del discurso", en F. Newmeyer (ed.), Panorama de la Lingüistica Moderna de la Universidad de Cambridge, vol. IV: El lenguaje: contexto socio-cultural, Madrid, Visor, págs. 275-298.

BorRego NiETO, J. (dir.) (2013): Gramática de referencia para la enseñanza de español. La combinación de oraciones, Salamanca, Universidad de Salamanca.

Briz, A., Pons Bordería, S. y Portolés, J. (2004): Diccionario de partículas discursivas del español. Publicación electrónica: <www.dpde.es>.

Brown, P. y Levinson, S. (1978): "Universals in language usage: Politeness phenomena”, en E. Goody (ed.), Questions and Politeness: Strategies in Social Interaction, Cambridge, Cambridge University Press, págs. 56-290.

Calsamiglia Blancafort, H. y Tusón Valls, A. (1999): Las cosas del decir. Manual de análisis del discurso, Barcelona, Ariel.

Domínguez García, N. (2002a): La organización del discurso argumentativo. Los conectores, Salamanca, Universidad de Salamanca.

- (2002b): "La polivalencia de pues: comportamiento argumentativo y metadiscursivo", en IV Congreso de Lingüistica General, Cádiz, del 3 al 6 de abril de 2000, vol. 2, págs. 811-820.

EsCANDEll Vidal, M. V. (1996): Introducción a la pragmática, Barcelona, Ariel.

Fuentes Rodríguez, C. (2009): Diccionario de conectores y operadores del español, Madrid, Arco/Libros.

GARCÉs Gómez, M. P. (2008): "El discurso y los marcadores", en La organización del discurso: marcadores de ordenación y de reformulación, Madrid, Iberoamericana, págs. 13-34.

- (2013): Los adverbios con función discursiva. Procesos de formación y evolución, Madrid/Franfurt, Iberoamericana/Vervuert.

GonZÁlez Ruiz, R. y Llamas SAíz, C. (eds.) (2011): Gramática y discurso: Nuevas aportaciones sobre partículas discursivas del español, Barañáin, Universidad de Navarra.

Haverkate, H. (1994): La cortesía verbal: estudio pragmalingüistico, Madrid, Gredos.

Hernando Cuadrado, L. (1994): "Sobre el funcionamiento de pues en el enunciado discursivo", en Actas del V Congreso ASELE. Publicación electrónica en: $<$ http://cvc.cervantes.es/ensenanza/biblioteca_ele/asele/pdf/05/05_0041.pdf $>$.

Holgado Lage, A. (2014): "Pragmática Lingüística Aplicada al Español para Extranjeros, con especial atención a los Marcadores Discursivos: El Diccionario de Marcadores Discursivos para Estudiantes de Español como Lengua Extranjera”, Tesis doctoral, Salamanca, Universidad de Salamanca. 
LANDONE, E. (2009): Los marcadores del discurso y la cortesía verbal en español, Berna, Peter Lang.

Loureda, Ó. y Acín, E. (eds.) (2010): Los estudios sobre marcadores del discurso en español, hoy, Madrid, Arco/Libros.

Mancera Rueda, A. (2009): "Los marcadores discursivos del coloquio", en Oralización de la prensa española: la columna periodística, Berna, Peter Lang, págs. 142-178.

Martín ZorraQuino, M. A. (2004): "El tratamiento lexicográfico de los marcadores del discurso y la enseñanza de E/LE”, en Actas del XV Congreso Internacional de ASELE, Sevilla, págs. 53-70.

- (2011): "El tratamiento de los marcadores del discurso en la Nueva Gramática de la Lengua Española (NGLE) de la Real Academia Española”, en J. J. de Bustos Tovar, R. Cano Aguilar, E. M. García de Paredes y A. López Serena (coords.), Sintaxis y análisis del discurso hablado en español. Homenaje a Antonio Narbona, Sevilla, Universidad de Sevilla, vol. 2, págs. 843-864.

- y Montolío Durán, E. (coords.) (1998): Los marcadores del discurso. Teoría y análisis, Madrid, Arco/Libros.

- y Portolés, J. (1999): "Los marcadores del discurso", en I. Bosque y V. Demonte (dirs.), Gramática Descriptiva de la Lengua Española, vol. 3, Madrid, Espasa, págs. 4051-4207.

MonTES, R. (1999): "The development of discourse markers in Spanish: interjections”, en Journal of Pragmatics, 31, págs. 1289-1319.

PÉrez-SalazAr, C. (2009): "Ni hablar, ni pensar, ni soñar. Análisis histórico de su transformación en unidades fraseológicas", en Nueva Revista de Filología Hispánica, LVII (1), págs. 37-64.

Perona, J. (2000): "La cohesión textual y los enlaces extraoracionales", en M. Alvar (dir.), Introducción a la lingüística española, Barcelona, Ariel, págs. 445-462.

PortolÉs, J. (1989): "El conector argumentativo pues", en Dicenda, 8, págs. 117133. Publicación electrónica en: <dialnet.unirioja.es/servlet/articulo?codigo $=90780>$.

- (1998): Marcadores del discurso, Barcelona, Ariel.

- (2008): "Las definiciones de las partículas discursivas en el diccionario", en M. P. Garcés Gómez (ed.), Diccionario histórico: nuevas perspectivas lingüísticas, Madrid, Iberoamericana, págs. 179-202.

Real ACAdemia Española, Banco de datos (CORDE): Corpus diacrónico del español. disponible en: <http://www.rae.es/recursos/banco-de-datos/corde>, consultado el 15-09-2015.

- (CORPES XXI): Corpus del español del siglo XXI. Disponible en: <http://www.rae. es/recursos/banco-de-datos/corpes-xxi>, consultado el 15-09-2015.

- (CREA): Corpus de referencia del español actual. Disponible en: <http://www. rae.es/recursos/banco-de-datos/crea>, consultado el 15-09-2015.

- y Asociación de Academias de la Lengua Española (2005): Diccionario panhispánico de dudas, Bogotá, Santillana.

- (2010): Nueva gramática de la lengua española. Manual, Madrid, Espasa-Calpe.

- (2001): Diccionario de la lengua española, 22a ed., Madrid, Espasa.

- (2014): Diccionario de la lengua española, $23^{\mathrm{a}}$ ed., Madrid, Espasa. 
RoJAS, E. (2011a): "La metáfora visual y el control del contacto. Indicadores de complejidad sistémica en la fijación de partículas conversacionales", en Actas de la I Jornada de Jóvenes Lingüistas, Instituto de Lingüística, Facultad de Filosofía y Letras, Universidad Nacional de Buenos Aires.

- (2011b): “¡Posta! y ¡De una! Marcadores de modalidad epistémica en la variedad juvenil del español argentino metropolitano: Un estudio contrastivo”, en Actas del II Coloquio Internacional Marcadores discursivos en las lenguas románicas. Un enfoque contrastivo, Instituto de Lingüística, Facultad de Filosofía y Letras, Universidad de Buenos Aires, págs. 205-218.

- (2012): "Algunos aportes de la pragmática inferencial a la reflexión metalingüística en torno a los marcadores del discurso: una experiencia didáctica en formación docente sobre la variedad juvenil del español metropolitano”, en Actas del VIII Congreso Internacional Orbis Tertius, Facultad de Humanidades y Ciencias de la Educación, Universidad Nacional de La Plata.

SAn Martín NúÑEZ, A. (2013): "Los reformuladores de distanciamiento en el corpus PRESEEA de Santiago de Chile", en Boletín de Filología de la Universidad de Chile, 48:1, págs. 171-199.

SÁnchez Carranza, A. (2013): "An analysis of pues as a sequential marker in Mexican Spanish talk-in-interactions”, en Spanish in Context, 10:2, págs. 284309.

SAntos Río, L. (2003): Diccionario de Partículas, Salamanca, Luso-Española de Ediciones.

SPERBER, D. y WiLSON, D. (1986): “Sobre la definición de Relevancia”, en L. Valdés Villanueva (ed.), La búsqueda del significado, Madrid, Tecnos, págs. 583-598.

Uribe Mallarino, M. (2005): "Conectores y contrastividad, el caso de pues", en AISPI Actas XXIII. Publicación electrónica disponible en: <http://cvc.cervan tes.es/literatura/aispi/pdf/22/II_35.pdf >.

VÁZQUez VeigA, N. (1994-1995): "Una aproximación a algunos marcadores con función textual de 'resumen', 'conclusión' y 'cierre'”, en Estudios de Lingüística, 10, págs. 349-390.

- (2003): Marcadores discursivos de recepción, Santiago de Compostela, Universidade de Santiago de Compostela. 